\title{
THE OPTICAL IDENTIFICATION OF RADIO SOURCES
}

\author{
D. W. DEWHIRST \\ The Observatories, University of Cambridge, England
}

Previous attempts to identify any large proportion of the discrete sources discovered at meter wavelengths have met with small success. In the investigation briefly reported here an extensive search has been made on the original plates of the 48-inch Palomar-National Geographic Society Sky Survey, using the available published radio data, but more especially the as yet unpublished results of a survey between +50 and -10 degrees declination that has been made with the interferometer of the Mullard Radio Astronomy Observatory, Cambridge. This radio survey $(3 \mathrm{C})$ has been carried out at $159.5 \mathrm{Mc} / \mathrm{s}$ using the aerial array of the $2 \mathrm{C}$ survey [1] in modified form. An account of the observation and reduction of this recent survey is given by other speakers in the Symposium. The area of sky covered by the $3 \mathrm{C}$ survey, and the criteria for the selection and classification of the sources, are likely to undergo small extensions and modifications before the final catalog is ready for publication, but these modifications will be of a minor character and will not alter the general conclusions of the present paper.

\section{THE AVAILABLE RADIO MATERIAL}

A preliminary radio survey of the sky in the area between $0^{\mathrm{h}}$ and $8^{\mathrm{h}}$ and the declination limits +10 and -20 degrees was made by Mills and Slee [2] with a pencil-beam instrument at $85.5 \mathrm{Mc} / \mathrm{s}$. These authors pointed out the gross discrepancies between their survey and $2 \mathrm{C}$ in this area. A revision of the pencil-beam survey in this area and an extension of the reduction through the whole zone between declinations +10 and -20 degrees has now been completed, and these authors have also kindly supplied details in advance of publication.*

In reducing the $3 \mathrm{C}$ observations the sources have been classified as $A, B$, and $C$ in order of reliability. The $A$ sources are clear and consistent on the the records either because of their intensity or because of an evident absence of confusion; it is believed that they are all real and not more than 10 per cent subject to an uncertainty in distinguishing the central right ascension lobe. At the other extreme the $C$ sources may well be misinterpretations of the records, and on internal evidence some 50 per cent are likely to be fictitious; of the real sources many will be placed in the wrong lobe of the interferometer pattern. The $C$ sources have been deduced from the records only in limited areas of the sky, and especially in the area $0^{\mathrm{h}}$ to $8^{\mathrm{h}}$ between

* Published as: Mills, B. Y., Slee, O. B., and Hill, E. R. Aust. J. Phys. 11, 360, 1958. 
+10 and -10 degrees to permit comparison with the pencil-beam survey. It is not intended to include these $C$ sources in the final catalog. In the selected area between $0^{\mathrm{h}}$ and $8^{\mathrm{h}}$ a total of $1673 \mathrm{C}$ sources are listed; there are 225 sources in the final Sydney catalog in the same area. In the whole $3 \mathrm{C}$ catalog between declinations +50 and -10 degrees there are listed $93 A$ sources and $397 B$ sources; taking the area of the primary reception beam of a single element of the interferometer to be approximately 8 square degrees at 159.5 $\mathrm{Mc} / \mathrm{s}$, there are 26 primary beam areas per source for the $A$ sources and 5 beam areas per source for the $B$ sources. Using this simplified criterion as a measure of confusion, it is clear that the confusion will be considerably less than for the $2 \mathrm{C}$ catalog, for which the corresponding figure is 0.5 beam areas per source for the total of 1936 sources listed in the catalog.

\section{COMPARISON OF RADIO SURVEYS}

In view of the radio difficulties we should wish to look especially, in an optical search program, at those positions for which there is agreement between radio surveys carried out by different methods. Only partial data were available at the time the optical study was carried out, but we can now assess the reliability of the positions used by examining the agreement in the overlap region between the Sydney and Cambridge surveys. It is difficult to establish rigorous criteria for defining "coincidences" in making a comparison of two radio surveys and we do not discuss this problem here. It is necessary, for example, to bear in mind the different nature of the two aerial arrays, which will respond differently to the same situation in the sky even at intensity levels where confusion is not a serious problem; thus, where a source is extended, it may be justifiable to assume that the same object is being observed by both pencil-beam and interferometer even though the agreement in position and intensity is apparently poor. At the intensity levels quoted for most of the $3 C B$ sources it is unlikely that the pencil-beam survey is subject to serious confusion except in regions of high and irregular background in low galactic latitude. The pencil-beam survey can therefore be used to determine the existence of lobe ambiguities. On the other hand, the very high resolving power of the interferometer in right ascension gives, for individual intense sources, more reliable right ascensions than can be determined with a pencil-beam instrument. In the following comparisons the criterion for agreement between individual sources is based on a method of " informed inspection," using all the available radio data in the region to decide whether the same physical object is being observed. The regions are: (1) the $B$ and $C$ sources in the area $0^{\mathrm{h}}$ to $8^{\mathrm{h}}$ between +10 and -10 degrees; (2) the $A$ sources in the region $8^{\mathrm{h}}$ to $24^{\mathrm{h}}$ between the same declinations. The small selected area which has been intensively studied contains no sources of class $A$.

It should be noted that the selected area between $0^{\mathrm{h}}$ and $8^{\mathrm{h}}$ would be expected to give almost the worst result on comparing the two surveys: it is small enough to be fortuitously deficient in small-diameter intense sources, and also 
TABLE I

A COMPARISON OF SURVEYS

$3 C$ sources

in regions
$(1)$ and (2)

(1) $44 B$ sources $123 C$ sources

(2) $20 A$ sources

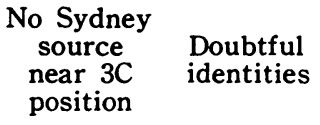

14

72

2

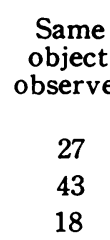

Fit after Fit after R. A. lobe dec. lobe shift to shift to

$\begin{array}{cc}10^{*} & 5 \\ 22^{*} & 8 \\ 1 & \text { None }\end{array}$

* An uncertain number of these situations may be "direct" fits, since the pencilbeam right ascensions are probably not always accurate enough to distinguish between the 10-minute-of-arc spacing of the interferometer lobes.

contains a large region in low galactic latitude near the Orion complex, where both surveys are subject to misinterpretation for different reasons. The much poorer agreement near the plane is obvious on inspection of radio maps of the region. In the last comparison, of the $A$ sources, the number of exact correspondences should be further increased by one: further examination of the Sydney records shows an intense source in the position of $3 \mathrm{C} .273$ (near Virgo A) that had previously been rejected as a Virgo sidelobe but is clearly real. This source is at right ascension $12^{\mathrm{h}} 26^{\mathrm{m}} 44^{\mathrm{s}}$, declination $+02^{\circ} 22^{\prime}$; it appears also on Westerhout's survey at $1390 \mathrm{Mc} / \mathrm{s}$. The one $3 \mathrm{C}$ source for which there is no corresponding Sydney source is in a very confused region in the plane at $l=11^{\circ}, 3 \mathrm{C} .398=2 \mathrm{C} .1630$ at $19^{\mathrm{h}} 08^{\mathrm{m}} 45^{\mathrm{s}},+9$ degrees 08 minutes of arc (corrected $3 \mathrm{C}$ position). The agreement between the Cambridge and Sydney observations of the $A$ sources is very satisfactory, and justifies the assumption that the $A$ sources of $3 \mathrm{C}$ are the most reliably determined sources of the northern sky. In the present paper the optical studies of these positions only are described.

\section{SYSTEMATIC AND RANDOM ERRORS OF POSITIONS}

A preliminary comparison shows a negligibly small systematic error between the right-ascension systems of the two surveys. Comparison of the 3C "raw" declinations with other radio positions and with previously known optical identifications showed a systematic error in the sense $3 \mathrm{C}$ north of true position by amounts that increase from 2 minutes of arc at +50 degrees to 23 minutes of arc at -10 degrees, and the declinations were corrected accordingly. The correction curve used is found to agree well at low declinations with that derived independently by comparing the 3C $A$ sources with the listed Sydney positions. After the correction has been applied, 13 of the 18 correspondences have declination differences of less than 6 minutes of arc; the outstanding errors (up to 25 minutes of arc) are associated with suspected extended sources that also show flux discrepancies. The $A$ sources have reading errors in right ascension quoted between $\pm 2^{\mathrm{s}}$ and $\pm 8^{\mathrm{s}}$, the straight arithmetic mean of the quoted errors is \pm 4.7 . The corresponding values in declination are \pm 2 minutes of arc, \pm 15 minutes of arc, mean \pm 6 minutes 
TABLE II

\section{Optical IDENTIFICATIONS With 3C $A$ SOURCES}

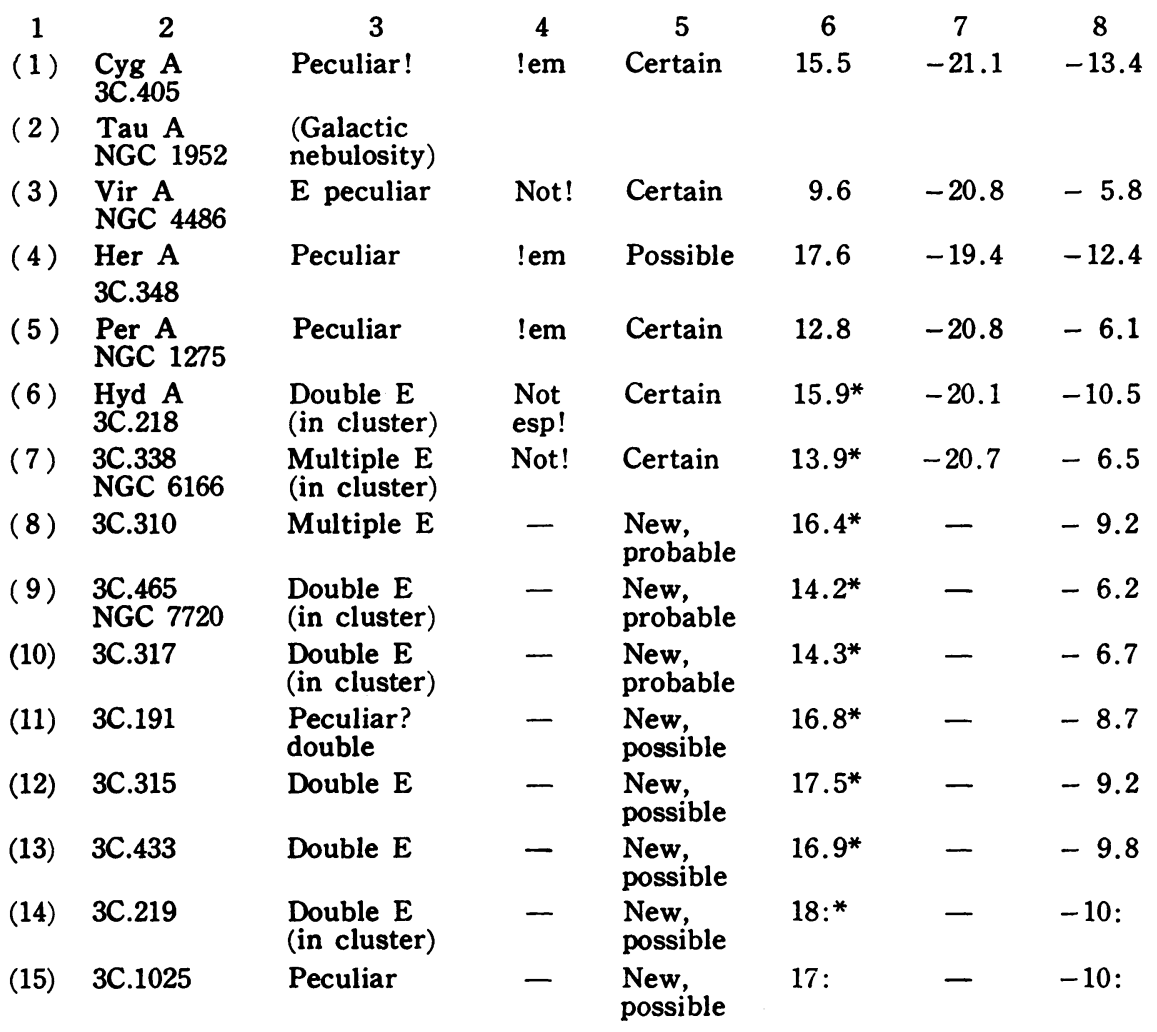

* Integrated magnitude of components.

\section{Notes}

(4) A faint galaxy with pronounced emission spectrum, studied by Minkowski. It has long been suspected as the source Hercules $A$ because of its peculiar nature. The observed radio positions of both Sydney and $3 C$ are in excellent agreement and both precede the galaxy by an amount that seems greater than the error of the radio positions. The positions fit better with a still fainter galaxy, not studied, or alternatively a second unresolved radio source may be causing confusion. The identification should not be regarded as certain.

(6) Previously regarded as uncertain because the object is not especially remarkable, the more accurate radio positions of Sydney and $3 \mathrm{C}$ now confirm the identity. The system consists of two rather widely separated galaxies, the brighter of the pair itself containing two nuclei inside a common envelope. In addition to $\lambda 3727$ the spectrum also shows the nebulium lines and $\mathrm{H} \beta$, weak and broad.

(7) The object is only $1^{\mathrm{s}} f$ and $1^{\prime} .5 S$ of the radio position, which has quoted errors of $\pm 5^{\mathrm{s}}, \pm 2$ minutes of arc. Like the Hydra A object NGC 6166 is also the brightest member of a compact cluster, and contains within its envelope two or possibly three nuclei in addition to that of the main system, which should perhaps be classed as an So. Spectra by Minkowski show only $\lambda 3727$ in the nucleus of the main component, but the velocity difference between the two brightest components is about $1500 \mathrm{~km} / \mathrm{sec}$ ond. If they are true cluster members, the other galaxies in the cluster show high velocity dispersion with velocities ranging at least from +7600 to $+9200 \mathrm{~km} / \mathrm{second}$. 
of arc. All the known optical and new "probable" identifications lie within the quoted errors for the corresponding source. For all the "direct" correspondences between the Cambridge $A$ and the Sydney sources, the agreement in right ascension is within the quoted probable errors of the listed positions.

\section{OPTICAL IDENTIFICATIONS}

Table II summarizes the data on the known and proposed new identifications with $A$ sources of 3C. The columns are as follows: (1) running number, (2) provisional $3 \mathrm{C}$ designation or other identities, (3) nature of object (all are galaxies except Taurus A), (4) note on spectrum, (5) status of identification, (6) apparent photographic magnitude, (7) absolute photographic magnitude, calculated for objects of known radial velocity assuming $H=100 \mathrm{~km} / \mathrm{second} /$ megaparsec, (8) $m_{1.9}-m_{p g}$.

The spectra are distinguished as !em indicating that there are numerous emission lines, or not! indicating no emission features other than 23727 , which is not unusual in E galaxies. For the well known objects $m_{p o}$ is taken from the recent literature; for the other objects approximate values have been determined on the Sky Survey plates, using a comparison image sequence of photored magnitudes kindly made available by G. O. Abell. The red magnitudes derived have been transformed to $m_{p q}$, and all magnitudes further corrected for extinction and to $b=90$ degrees, using the data of Abell [3] and Holmberg [4]. The radio magnitudes have been derived by reducing the available data to a common system at $158 \mathrm{Mc} / \mathrm{s}$ using a known or assumed spectral index, following Whitfield [5]. Both sets of magnitude data clearly leave much to be desired and the decimal plane deserves little significance, but the systems should be internally consistent.

Brief notes follow the table.

\section{DISCUSSION}

All of the galaxies designated as double or multiple $\mathrm{E}$ in the above table require further investigation. On the scale of the Sky Survey plates it is difficult to distinguish between $\mathrm{E}$ and S0 galaxies, but it seems certain that all the systems lack spiral arms or dust. The most striking result of the investigation, if the identifications are tenable, is that we recognize for the first time a new type of object which tends to be a source, namely close double or multiple $\mathrm{E}$ galaxies, usually sufficiently close to share a common outer extended envelope, and occurring either as the brightest members of small groups of E and S0 galaxies (as distinct from rich extended clusters), or as field objects. It is tempting to suppose, pending the collection of further spectroscopic information, that we are dealing with radio emission associated with a particular phase in the development of small groups of $E$ and S0 galaxies. The systems appear to be physically distinguishable from such "freak" objects as NGC 5128 and NGC 1275, characterized by their rich emission spectra and the presence of irregular dust patches. 
The listed probable error areas of the $A$ sources are about 25 square minutes of arc, so that we should expect to find a normal galaxy of $18^{\mathrm{m}}$ in about onethird of the listed places. The optical magnitudes of numbers 6 to 10 in the above list are such that even as single normal galaxies we should not expect them to be chance coincidences; their similar nature as multiple systems lends strength to these identifications. Such multiple galaxies are common objects on the Sky Survey plates at fainter magnitudes, and because of this the objects numbered 11 to 15 must be accepted as identifications with greater reserve pending further study. A preliminary count reduction of double and peculiar galaxies to various magnitude limits on Sky Survey plates (Dewhirst, unpublished) suggests that they are significant coincidences. The question remains why these particular systems should be sources when numerous apparently similar objects in the sky are not proportionally strong emitters.

In addition to the objects listed above there are 6 other normal galaxies close to source positions that are bright enough to be better than chance coincidences; it is possible that some of these could be more distant objects of the NGC 4486 type that would be unrecognizable as peculiar on the plates. The survey confirms the results of earlier investigations, that no objects other than galaxies or galactic nebulosities can be identified, and that the bright well-known peculiar galaxies are not, as a class, intense emitters.

Unidentified sources. - Fourteen of the sources lie in low galactic latitude where galaxies would be difficult to observe; careful scrutiny has revealed no new galactic objects in these positions. There are some 50 positions where no recognizably peculiar galaxy brighter than $18^{\mathrm{m}}$ can be found acceptably close to the radio position.

A powerful argument for the extragalactic nature of these unidentified objects could come from measures of radio diameters, but the present available radio data on diameters conflict. Three of the examined but unidentified sources have radio diameters of less than 12 seconds of arc according to Morris, Palmer, and Thompson [6]. Assuming that the radio diameter can be larger than the maximum optical diameter by a factor of 4 or 5 (e.g., Cygnus A or Virgo A) the optical objects would be less than 3 seconds of arc diameter. The evidence in column 7 of Table II, and the values for such southern galaxies as NGC 1316 and NGC 5128, suggest that the identified source galaxies are among those of highest known luminosity. Even such galaxies would be fainter than $21^{\mathrm{m}}$ at a distance corresponding to an angular size of 3 seconds of arc. On the other hand there are three further sources for which diameters are quoted in the literature at $4 \frac{1}{3}, 5$, and 6 minutes of arc, where no optical identification can be made. A galaxy with an optical diameter of the order of one minute of arc should not be fainter than $16^{\mathrm{m}}$, thus being readily recognizable. Assuming the reliability of the positions and diameters, this discrepancy could be explained by assuming: (1) that the measured diameters are not true diameters but represent close double sources or more complex intensity distributions, (2) that galaxies exist that have radio dimensions much greater than that of an object like Cygnus A, or (3) that these situations represent sources that are not of extragalactic nature. While 
the bulk of the evidence favors one of the former explanations, an extensive study of the intensity distributions across the 3C $A$ sources is perhaps as important as accurate positions if we are to elucidate the nature of the still unidentified sources.

The investigations reported above will be described in detail elsewhere: they are a part of the ones I carried out when I was a guest investigator at Mount Wilson and Palomar Observatories, and a visitor at the Division of Radiophysics of C.S.I.R.O., Sydney. I am grateful for financial support from a National Science Foundation Fund administered by the Carnegie Institution, from the Royal Society, and from the Commonwealth Scientific and Industrial Research Organization. Dr. R. Minkowski and the several radio astronomy groups have at all times kindly placed their unpublished observations at my disposal.

\section{REFERENCES}

[1] Shakeshaft, J. R., et al. Mem. R.A.S. 67, 97, 1955.

[2] Mills, B. Y., and Slee, O. B. Aust. J. Phys. 10, 162, 1957.

[3] Abell, G. O. Ap. J. Suppl. Ser. 3, 211, 1958.

[4] Holmberg, E. Medd. Lunds. Astr. Obs. Ser. II, No. 136, 1958.

[5] Whitfield, G. R. M.N.R.A.S. 117, 680, 1957.

[6] Morris, D., Palmer, H. P., and Thompson, A. R. The Observatory, 77, 103, 1957.

\section{Discussion}

Lovell: Are there any angular-diameter measurements of the radio sources in these new identifications? If so, what is the relation between the radio and optical diameters?

Dewhirst: Radio diameters are available for a few of the sources that have been tentatively identified with optical objects, in addition to the wellknown identified objects that have already been studied. The radio observations of diameter do not agree very well, but on the whole the radio objects are of the same size as, or rather larger than, the optical objects, and there are no serious discrepancies between radio and optical angular diameter.

Minkowski: The curious fact should be noted that Mills finds a certain number of possible identifications with bright normal galaxies, while north of -10 degrees virtually no coincidences have been found by Minkowski or Dewhirst, using both Sydney and Cambridge positions. This seems to be a good example of an unlikely situation that can arise from too small a sample. 